\title{
Resveratrol protects against high glucose-induced oxidative damage in human lens epithelial cells by activating autophagy
}

\author{
PENGZHI CHEN ${ }^{1}$, ZHENYU YAO ${ }^{2}$ and ZHIHUI $\mathrm{HE}^{2}$ \\ ${ }^{1}$ Department of Ophthalmology, The Affiliated Hospital of Inner Mongolia University for Nationalities; \\ ${ }^{2}$ Medical College, Inner Mongolia University for Nationalities, Tongliao, Inner Mongolia 028006, P.R. China
}

Received October 22, 2019; Accepted July 1, 2020

DOI: $10.3892 /$ etm.2021.9871

\begin{abstract}
In the pathogenesis of diabetic cataract, high glucose levels induce oxidative damage in human lens epithelial cells (HLECs). Resveratrol has been demonstrated to be a potent antioxidant in various disease conditions; however, limited information is available on its effects on oxidative damage associated with the pathogenesis of diabetic cataract in HLECs. The present study aimed to determine whether resveratrol prevents high glucose-induced oxidative damage to human lens epithelial cells by activating autophagy. In the present study, HLECs treated with high glucose were used as a cellular model of diabetic cataract and treated with resveratrol for $24 \mathrm{~h}$. Flow cytometry was performed to detect the cellular reactive oxygen species (ROS) content. Autophagy marker protein levels were determined by western blotting. Immunofluorescence assay was performed to analyze in vitro microtubule-associated protein 1 light chain $3 \beta$ (LC3B) protein expression. Autophagosome formation in HLECs was observed using transmission electron microscopy. The results demonstrated that high glucose suppressed HLEC viability and proliferation rate compared with normal glucose levels $(5 \mathrm{mM})$, which were significantly reversed by resveratrol treatment. High glucose also increased the ROS content compared with ROS content in normal HLECs, which was reduced following resveratrol treatment. Further experiments demonstrated that resveratrol significantly reversed the high glucose-decreased protein levels of LC3II and beclin-1 proteins and the high glucose-increased protein levels of LC3I and p62 in HLECs. In conclusion, resveratrol inhibited the high
\end{abstract}

Correspondence to: Dr Pengzhi Chen, Department of Ophthalmology, The Affiliated Hospital of Inner Mongolia University for Nationalities, 1742 Huolinhe Street, Horqin, Tongliao, Inner Mongolia 028006, P.R. China

E-mail: chenpengzhidor@163.com

Abbreviations: HLECs, human lens epithelial cells; TEM, transmission electron microscopy; LC3B, microtubule-associated protein 1 light chain $3 \beta$; MAPK, mitogen-activated protein kinase

Key words: resveratrol, autophagy, p38 mitogen-activated protein kinase signaling, diabetic cataract glucose-induced oxidative damage in HLECs by promoting autophagy through the activation of the p38 mitogen-activated protein kinase signaling pathway. These results provide a theoretical basis for the application of resveratrol in diabetic cataract prevention and treatment.

\section{Introduction}

Diabetic cataract is a debilitating ophthalmic disease and a major cause of visual impairment and blindness in both developed and developing countries with increased incidence and progression compared with other diabetes complications in patients with diabetes mellitus (1-3).In the UK, incidence rates of cataract were 20.4 (95\% CI, 19.8-20.9) per 1,000 person-years (py) in patients with diabetes and 10.8 (95\% CI, 10.5-11.2) per 1,000 py in the general population (1). Due to the increasing numbers of cases of diabetes mellitus worldwide, the incidence of diabetic cataract has steadily increased, especially in developing countries, due to limited accesses to cataract surgery $(3,4)$. A recent epidemiological study reported that diabetic cataract was also an early ocular complication in child and adolescent patients with diabetes mellitus (5). Although chataract surgery is widely applied for diabetic cataract treatment, the pathogenic mechanisms underlying cataract development in diabetes is still poorly understood, which makes it challenging to prevent diabetic cataract at its initial stages (4). In addition, a high possibility of complications from cataract surgery has also been observed in patients with diabetes mellitus, presenting a challenging medical issue (4). Thus, a complete understanding of the molecular pathogenic mechanisms of diabetic cataract may facilitate the development of novel modules for its prevention and treatment.

Previous studies have demonstrated that the pathogenic development of diabetic cataract is associated with abnormal metabolic regulation $(4,6,7)$. For instance, accumulation of sorbitol produced as a result of enzymatic reduction of glucose by aldose reductase, has been established as an initiating factor for diabetic cataract pathogenesis $(7,8)$. Specifically, the intracellular deposition of the polyol sorbitol causes significant osmotic changes in lens tissues, resulting in lens fiber degeneration and liquefaction, eventually inducing sugar cataract formation, lens swelling and opacities $(4,9)$. In addition, the apoptosis of lens epithelial cells (LECs) is also enhanced by the osmotic stress induced by abnormal sorbitol accumulation 
in the lens, which is also involved in diabetic cataract formation $(10,11)$. Normally, the mitochondrial oxygen consumption by human LECs (HLECs) performs crucial roles in lens metabolism and maintenance of a hypoxic environment around the lens, which is important for lens transparency (12). Under pathogenic conditions, high glucose effectively suppresses the proliferation and induces apoptosis and oxidative stress of HLECs, which is associated with lens opacities and cataract formation in diabetes (12-14). Therefore, the inhibition of LEC apoptosis and oxidative stress under high glucose conditions is regarded as a potential strategy for the prevention and treatment of diabetic cataract.

Autophagy refers to the cellular lysosomal degradation processes responsible for the degradation and recycling of abnormal proteins and damaged organelles, which is essential for cell survival and development, and is associated with various human diseases including diabetes mellitus $(3,15)$. The activation of the autophagy processes has been demonstrated to potentially suppress cell apoptosis and oxidative stress under specific conditions $(16,17)$. For instance, the activation of the autophagy process by eupatorine was demonstrated to suppress arachidonic acid and iron-induced oxidative stress in human liver cancer cells by alleviating mitochondrial dysfunction and inhibiting reactive oxygen species (ROS) production (16). Notably, autophagy has also been reported as a protective mechanism against oxidative stress in pancreatic $\beta$ cells and other insulin target tissues, including the skeletal muscles, adipose tissue and liver (15). In another recent report, a novel autophagy enhancer screened from a chemical library was effective in suppressing the metabolic syndrome and diabetes mellitus in a mouse model (18). Alterations of the autophagy process in HLECs have also been implicated in diabetic cataract progression (19); however, an effective regulator of autophagy in HLECs associated with the pathogenesis of diabetic cataract has not been established.

Resveratrol is a non-flavonoid polyphenol previously isolated from the grape seed (Vitis vinifera L.), which naturally occurrs as phytoalexin and is recommended for diabetic patients as a dietary supplement to control blood sugar levels in the body $(20,21)$. It has been documented in the past 10 years that resveratrol is beneficial in the prevention and treatment of various human disorders such as cancer, cardiovascular, Alzheimer's, inflammatory bowel and fatty liver diseases, as well as diabetes mellitus (20-22). In addition, resveratrol can also regulate cellular oxidative injury and autophagy processes under certain conditions; for instance, resveratrol suppressed the oxidative injury in human umbilical endothelial vein cells induced by oxidized low density lipoprotein by activating the silencing information regulator 1 (SIRT1) and modulating the AMP-activated protein kinase $\alpha 1$ signaling pathway (23). In addition, resveratrol has been recently demonstrated to induce autophagy in non-small-cell lung cancer cells by activating the p38 mitogen-activated protein kinase (MAPK) and inhibiting the Akt/mTOR signaling pathway (24). However, the effects of resveratrol on the autophagy and oxidative damage in HLECs associated with diabetic cataract pathogenesis are currently unknown.

The present study aimed to verify the hypothesis that resveratrol protects HLECs against high glucose-induced oxidative injury by enhancing the cellular autophagy process.

\section{Materials and methods}

Cell culture. HLECs were purchased from Procell Life Science \& Technology Co., Ltd. (cat. no. CP-H127) and cultured in Dulbecco's minimal essential medium (DMEM; cat. no. SH30022.01B; HyClone; Cytiva) supplemented with 10\% fetal bovine serum (FBS; cat. no. 10099-141; Gibco; Thermo Fisher Scientific, Inc.) and 5\% penicillin/streptomycin (Sigma-Aldrich; Merck KGaA) at $37^{\circ} \mathrm{C}$ in a humidified atmosphere with $5 \% \mathrm{CO}_{2}$.

Cell treatment. To establish the cellular model of oxidative stress, HLECs $\left(1 \times 10^{5}\right.$ cells/well) were plated into 6 -well plates and cultured at $37^{\circ} \mathrm{C}$ for $8 \mathrm{~h}$. The HLECs were then treated with $0,5,15,20,25$ or $30 \mathrm{mM}$ glucose (Sigma-Aldrich; Merck KGaA) for $24 \mathrm{~h}$ under normal culture conditions. Then, the glucose-treated $(25 \mathrm{mM})$ HLECs were treated with $0,1,2$ or $2.5 \mu \mathrm{M}$ resveratrol (cat. no. R5010; Sigma-Aldrich; Merck $\mathrm{KGaA}$ ) for $24 \mathrm{~h}$ at $37^{\circ} \mathrm{C}$ prior to subsequent experiments.

For MAPK inhibition, glucose-treated $(25 \mathrm{mM})$ and resveratrol-treated $(2 \mu \mathrm{M})$ HLECs were treated with $10 \mu \mathrm{M}$ BIRB 796 (cat. no. S1574; Selleck Chemicals) for 24 h at $37^{\circ} \mathrm{C}$ prior to subsequent experiments.

Cell viability. The viability of cultured HLECs was detected using the MTT Cell Proliferation and Cytotoxicity Assay kit (cat.no.C0009; Beyotime Institute of Biotechnology) according to the manufacturer's instructions. Briefly, the cultured HLECs were seeded into 96-well plates (2,000 cells/well) and cultured in DMEM with $10 \%$ FBS containing the specified glucose concentrations for $24 \mathrm{~h}$. Subsequently, cells in each well were incubated with $20 \mu \mathrm{l}$ MTT solution (cat. no. M-2128; Sigma-Aldrich; Merck KGaA) for $4 \mathrm{~h}$ under normal culture conditions, followed by incubation with $100 \mu \mathrm{l}$ DMSO for $4 \mathrm{~h}$ under normal culture conditions until the formazan crystals were completely dissolved. Finally, cell viability was determined by measuring the absorbance at $570 \mathrm{~nm}$ using a plate reader. At least three biological replicates were performed.

ROS content detection. The contents of ROS in the cultured HLECs were measured by flow cytometry using the Cell-based ROS/Superoxide Detection Assay kit (cat. no. ab139476; Abcam) according to the manufacturer's instructions. Briefly, HLECs after specified treatments were collected into $5 \mathrm{ml}$ round-bottom polystyrene tubes, washed with $1 \mathrm{X}$ Wash Buffer, resuspended in $500 \mu \mathrm{l}$ ROS/Superoxide Detection Solution and incubated for $30 \mathrm{~min}$ at $37^{\circ} \mathrm{C}$ in the dark. The ROS contents were determined using a flow cytometer (BD-C6, CFLOW plus 1.0, Becton, Dickinson and Company) equipped with a blue laser $(488 \mathrm{~nm})$. The ROS measurements were biologically repeated for at least three times.

Western blot assay. For the detection of protein levels in cultured HLECs, total proteins were extracted from cells after designated treatments using a RIPA lysis buffer (cat. no. 89900; Thermo Fisher Scientific, Inc.) according to the manufacturer's instructions. The total protein contents were determined by a bicinchoninic acid assay (cat. no. 23227; Thermo Fishier Scientific, Inc.). Subsequently, $20 \mathrm{mg}$ protein from each group 
A

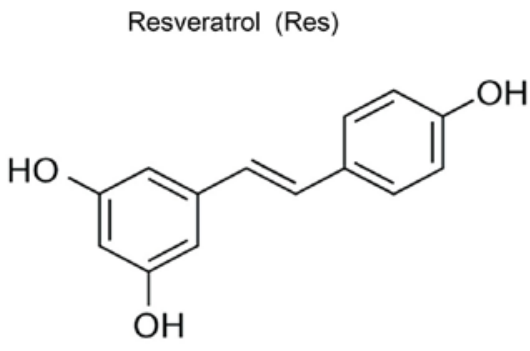

C

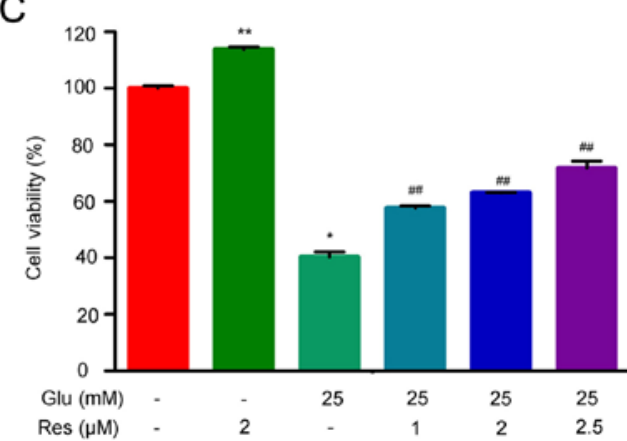

B

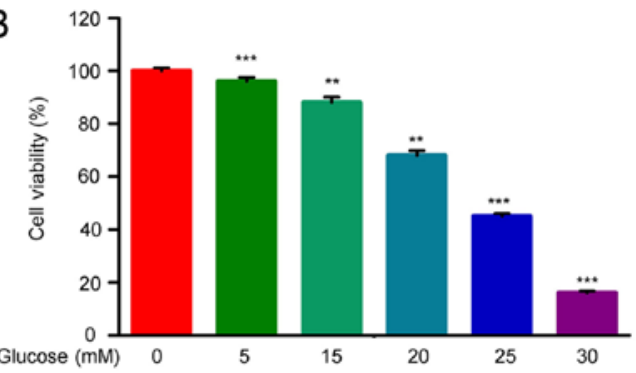

D

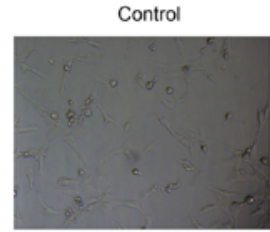

$\operatorname{Res}(2 \mu \mathrm{M})$

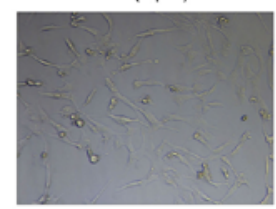

Glu $(25 \mathrm{mM})$

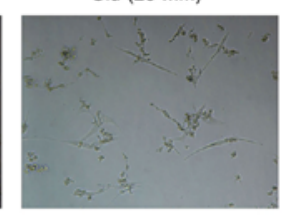

Glu $(25 \mathrm{mM})+\operatorname{Res}(2 \mu \mathrm{M})$

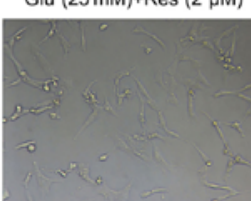

Figure 1. Resveratrol reverses high glucose-induced HLEC viability suppression. (A) Chemical structure of resveratrol. (B) The viability of HLECs treated with $0,5,15,2025$ or $30 \mathrm{mM}$ glucose for $24 \mathrm{~h}$ was measured by MTT assay. (C) HLEC viability following treatment with $25 \mathrm{mM}$ glucose and $0,1,2$ or $2.5 \mu \mathrm{M}$ resveratrol. (D) Morphological features of HLECs treated with $25 \mathrm{mM}$ glucose, $2 \mu \mathrm{M}$ resveratrol or the combination of $25 \mathrm{mM}$ glucose and $2 \mu \mathrm{M}$ resveratrol observed under an inverted microscope. ${ }^{*} \mathrm{P}<0.5,{ }^{* * *} \mathrm{P}<0.01$ and ${ }^{* * * *} \mathrm{P}<0.001$ vs. untreated control; ${ }^{* \#} \mathrm{P}<0.01$ vs. $25 \mathrm{mM}$ glucose alone. HLECs, human lens epithelial cells; Res, resveratrol.

was boiled at $100^{\circ} \mathrm{C}$ for $5 \mathrm{~min}$, separated by $10 \%$ SDS-PAGE and blotted onto PVDF membranes (EMD Millipore). The membranes were blocked with a $5 \%$ fat-free milk solution for $2 \mathrm{~h}$ at room temperature, incubated for $2 \mathrm{~h}$ at room temperature with primary antibodies appropriately diluted in TBS-1\% Tween-20, and incubated with diluted secondary antibodies $(1: 10,000)$ for $2 \mathrm{~h}$ at room temperature. The blots were visualized by developing with the ECL Western Blotting Substrate (cat. no. 32106; Thermo Fishier Scientific, Inc.). GAPDH protein levels were detected as the internal standard. The antibodies used were as follows: Anti-LC3-I/II (cat. no. ABC929; Sigma-Aldrich; Merck KGaA), anti-beclin-1 (cat. no. ab62557; Abcam), anti-P62 (cat. no. ab56416; Abcam), anti-p38 MAPK (cat. no. 8690; Cell Signaling Technology, Inc.), anti-phosphor-p38 MAPK (cat. no. 4511; Cell Signaling Technology, Inc.), anti-GAPDH (ab9485; Abcam) and HRP-conjugated goat anti-rabbit IgG (cat. no. ab205718; Abcam). At least three biological replicates were performed for protein level determination.

Immunofluorescence (IF) assay. The expression of LC3B protein in cultured HLECs was analyzed by IF for the evaluation of autophagy following the designated treatments. Briefly, cell slides were fixed with $4 \%$ paraformaldehyde for $15 \mathrm{~min}$ at room temperature and washed three times with PBS for $5 \mathrm{~min}$, followed by blocking with 3\% BSA (cat. no. ST025; Beyotime Institute of Biotechnology) for $1 \mathrm{~h}$ at room temperature, overnight incubation with primary antibodies against LC3B (1:200; cat. no. 2775; Cell Signaling Technology, Inc.) at $4^{\circ} \mathrm{C}$, three washes with PBS for $5 \mathrm{~min}$ and incubation with fluorophore-conjugated secondary antibodies (Alexa Fluor ${ }^{\circledR} 488$;
1:200; cat. no. ab150081; Abcam) for $2 \mathrm{~h}$ in the dark. The slides were finally mounted with the Prolong ${ }^{\circledR}$ Gold Antifade reagent (cat. no. 9071; Cell Signaling Technology, Inc.) and observed under a Leica DMI6000B fluorescence microscope (Leica Microsystems, Inc.). Three biological replicates were performed for analysis of LC3B expression by immunofluorescence.

Transmission electron microscopy. The formation of autophagosomes in HLECs was observed by transmission electron microscopy (TEM) as previously described (23). Briefly, HLECs grown on slides were fixed for $1 \mathrm{~h}$ with $2.5 \%$ glutaraldehyde (cat. no. G7776; Sigma-Aldrich; Merck KGaA) dissolved in $0.1 \mathrm{~mol} / 1 \mathrm{PBS}$ at room temperature, incubated with $1 \%$ osmium tetroxide (cat. no. O5500; Sigma-Aldrich; Merck $\mathrm{KGaA}$ ) for $30 \mathrm{~min}$ at room temperature and observed using a TecnaiG2 Spirit Bio TWIN transmission electron microscope (120 kV; FEI; Thermo Fisher Scientific, Inc.). The TEM experiments were performed at least three times.

Statistical analysis. Data are presented as the mean \pm SD of at least three biological replicates. The differences among groups were analyzed using the SPSS 20.0 software package (IBM Corp.) by one-way ANOVA followed by Tukey's post hoc test. $\mathrm{P}<0.05$ was considered to indicate a statistically significant difference.

\section{Results}

Resveratrol reverses the high glucose-induced suppression of $H L E C$ viability. To determine the potential regulatory functions of resveratrol (Fig. 1A) on HLEC viability associated with 

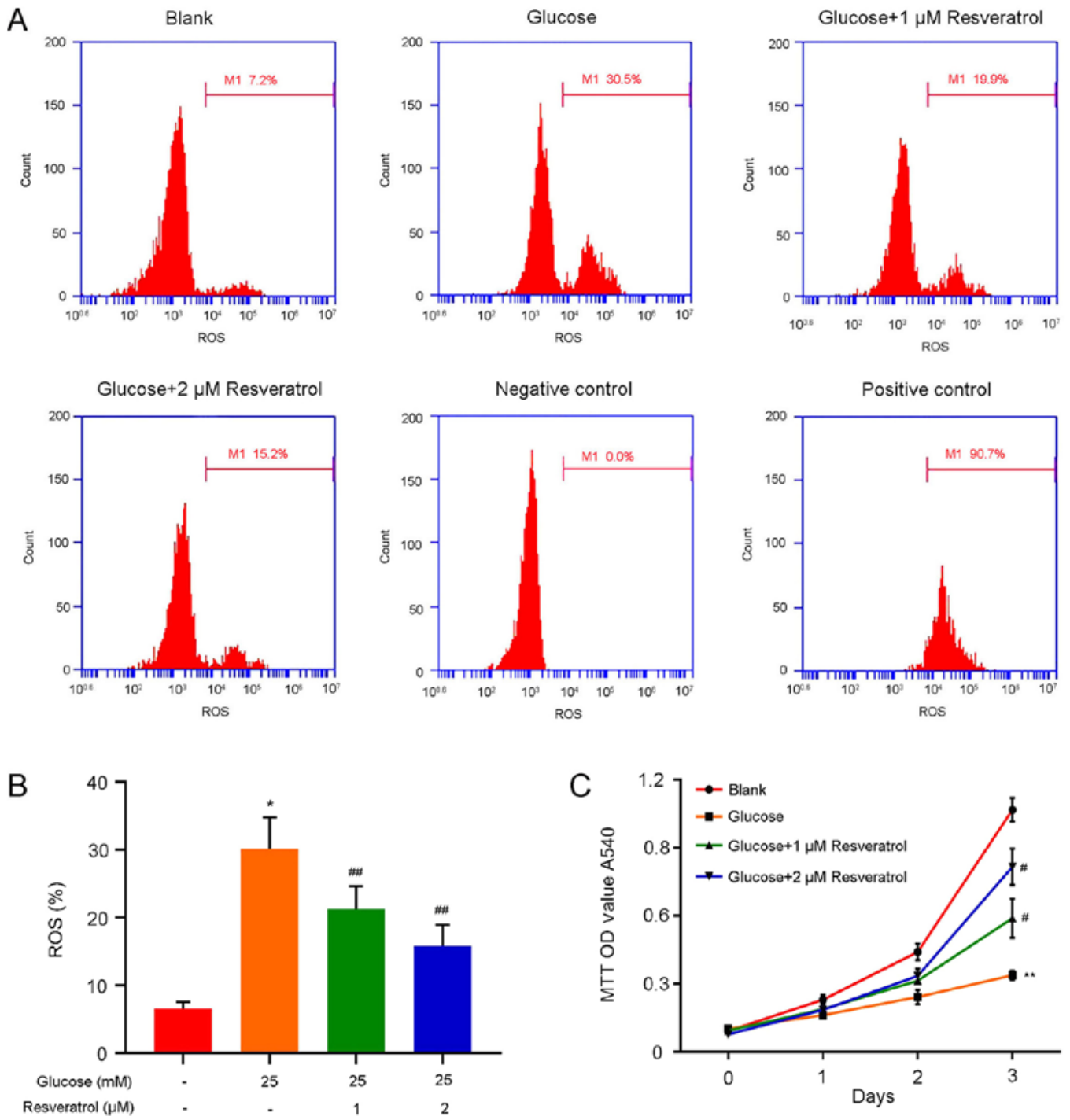

Figure 2. Resveratrol inhibits high glucose-induced HLEC oxidative damage. (A and B) ROS contents in HLECs treated with 25 mM glucose or the combination of $25 \mathrm{mM}$ glucose with 1 or $2 \mu \mathrm{M}$ resveratrol for $24 \mathrm{~h}$ were determined by flow cytometry. Untreated cells were used as the blank group. (C) The proliferation rates of HLECs treated with $25 \mathrm{mM}$ glucose or the combination of $25 \mathrm{mM}$ glucose with 1 or $2 \mu \mathrm{M}$ resveratrol were evaluated by the MTT assay after the designated treatments for 1,2 or 3 days. ${ }^{*} \mathrm{P}<0.05$ and ${ }^{* *} \mathrm{P}<0.01$ vs. untreated control; ${ }^{*} \mathrm{P}<0.05$ and ${ }^{\# \#} \mathrm{P}<0.01$ vs. 25 mM glucose alone. HLECs, human lens epithelial cells; ROS, reactive oxygen species.

diabetic cataract formation, the present study established the cellular model of HLEC oxidative stress by treating cultured HLECs with $0,5,15,20,25$ or $30 \mathrm{mM}$ glucose (Fig. 1B). Treatment with glucose concentrations $\geq 15 \mathrm{mM}$ resulted in a significant concentration-dependent decrease of HLEC cell viability (Fig. 1B). Subsequently, HLECs treated with $25 \mathrm{mM}$ glucose were treated with $0,1,2$ or $2.5 \mu \mathrm{M}$ resveratrol for $24 \mathrm{~h}$. The results demonstrated that the decreases in HLEC viability induced by high glucose were reversed by resveratrol treatment in a concentration-dependent manner (Fig. 1C). Treatment with $2.5 \mu \mathrm{M}$ resveratrol for $24 \mathrm{~h}$ increased the $25 \mathrm{mM}$ glucose-treated HLEC viability by $\sim 60 \%$ compared with that of HLECs treated with glucose alone (Fig. 1C). Microscopy revealed that $25 \mathrm{mM}$ glucose-treated HLECs lost their normal cell morphological features (the normal crystal epithelial cells are evenly distributed, with equal cell size and full shape and the cells are oval or polygonal), which were recovered by simultaneous treatment with $2 \mu \mathrm{M}$ resveratrol (Fig. 1D). These results demonstrated that resveratrol treatment effectively reversed the suppression of HLEC viability induced by high glucose.

Resveratrol mitigates the high glucose-induced oxidative damage in HLECs. High glucose-induced oxidative injury in HLECs mediates diabetic cataract pathogenesis (25). In the present study, the ROS contents in HLECs under combined treatment with high glucose and resveratrol were detected, and the results demonstrated that treatment with $25 \mathrm{mM}$ glucose induced a significant increase of ROS contents in HLECs compared with those in the blank group (Fig. 2A). However, treatment with 1 or $2 \mu \mathrm{M}$ resveratrol reduced the ROS contents in HLECs under high glucose treatment (Fig. 2A). In addition, the proliferation of HLECs under above conditions was detected; compared with that of untreated cells, the proliferation rate of HLECs was repressed by treatment with $25 \mathrm{mM}$ glucose, which was recovered by treatment with 1 or $2 \mu \mathrm{M}$ resveratrol (Fig. 2B). The alterations of the ROS contents and proliferation rates of HLECs suggested that resveratrol may 
A

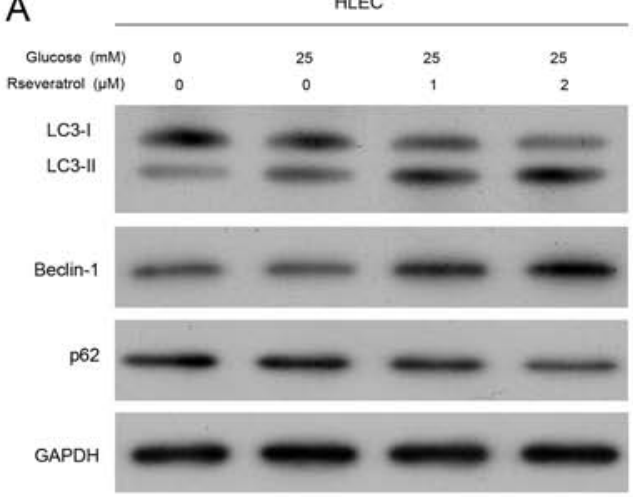

B
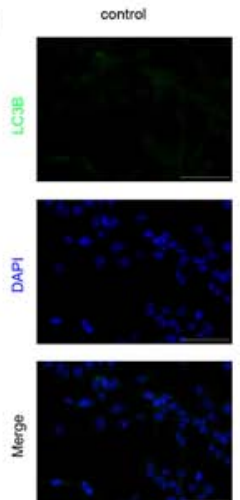
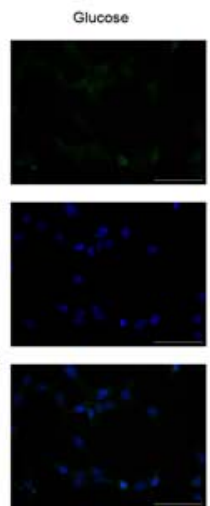

Glucose+1 $\mu \mathrm{M}$ Resveratrol
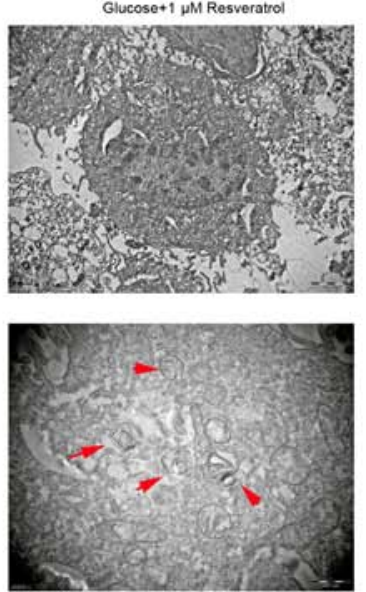

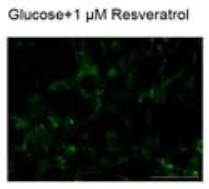

Glucose+2 2 M Rosoratol
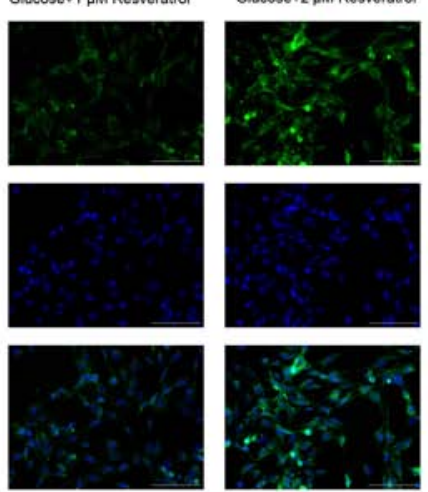

C
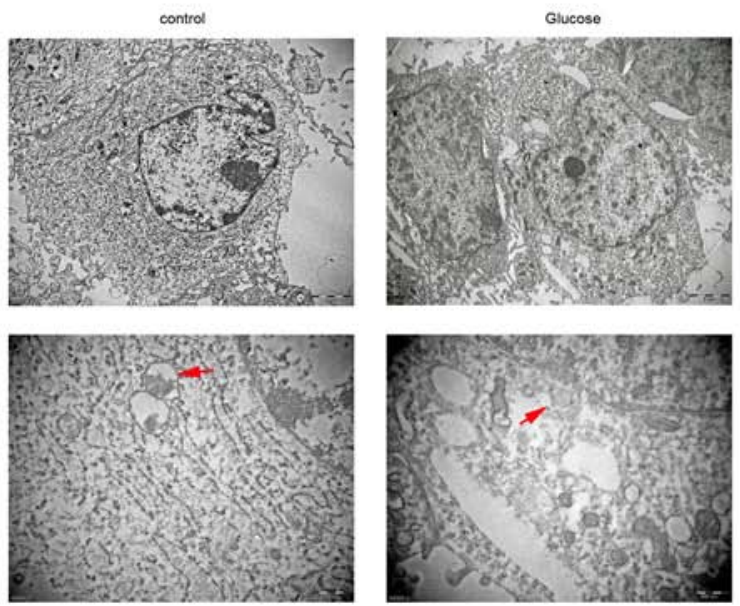

Figure 3. Resveratrol activates autophagy in HLECs under high glucose conditions. (A) Protein levels of autophagy markers LC3I, P62, LC3II and beclin-1 in HLECs treated with $25 \mathrm{mM}$ glucose and 1 or $2 \mu \mathrm{M}$ resveratrol for $24 \mathrm{~h}$ were determined by western blotting; GAPDH was used as the internal control. (B) Immunofluorescence detection of the expression of LC3B in HLECs treated with $25 \mathrm{mM}$ glucose and 1 or $2 \mu \mathrm{M}$ resveratrol for $24 \mathrm{~h}$. Cell nuclei were visualized by staining with DAPI. (C) Autophagosome formation in HLECs treated with $25 \mathrm{mM}$ glucose and 1 or $2 \mu \mathrm{M}$ resveratrol for 24 h was observed by transmission electron microscopy. Red arrows indicate autophagosomes. HLEC, human lens epithelial cell; LC3, microtubule-associated protein 1 light chain 3 .

act as an inhibitor of high glucose-induced oxidative damage in HLECs.

Resveratrol promotes autophagy in HLECs under high glucose. To explore the cellular processes underlying the suppression of high glucose-induced HLEC oxidative damage by resveratrol, the autophagy marker expression in HLECs treated with high glucose and resveratrol was subsequently determined. The protein expression of four autophagy markers was first detected by western blotting, which demonstrated that LC3I and P62 protein levels in cultured HLECs under high glucose treatment were reduced by 1 or $2 \mu \mathrm{M}$ resveratrol in a concentration-dependent manner (Figs. 3A and S1). By contrast, the protein expression levels of LC3II and beclin-1 in HLECs under high glucose conditions were elevated by 1 or $2 \mu \mathrm{M}$ resveratrol treatment (Fig. 3A). The IF assay demonstrated that the levels of LC3B protein in HLECs under high glucose were increased by treatment with 1 or $2 \mu \mathrm{M}$ resveratrol compared with those in untreated cells or cells treated with high glucose alone (Fig. 3B). In addition, the formation of autophagosomes in HLECs were analyzed by TEM. The results demonstrated that a limited number of autophagosomes were formed in untreated HLECs or those treated with high glucose alone; however, a large number of autophagosomes was observed in HLEC cells treated with high glucose and 1 or $2 \mu \mathrm{M}$ resveratrol (Fig. 3C). These results suggested that resveratrol treatment effectively activated autophagy in HLEC cells under high glucose treatment.

Resveratrol activates the p38 MAPK pathway to induce autophagy in HLECs under high glucose. The p38 MAPK signaling pathway is involved in autophagy development (26). The results of the present study demonstrated that the total p38 protein levels in HLECs treated with high glucose and $2 \mu \mathrm{M}$ resveratrol were decreased compared with those in untreated cells or cells treated with glucose alone (Fig. 4A). By contrast, the phosphorylated p38 protein levels HLEC cells under high glucose treatment were elevated by treatment with $2 \mu \mathrm{M}$ resveratrol for $24 \mathrm{~h}$ (Fig. 4A). These alterations of $\mathrm{p} 38$ expression and phosphorylation induced by resveratrol were reversed by the co-treatment with $10 \mu \mathrm{M}$ MAPK inhibitor BIRB796 (Fig. 4A). Notably, IF results also confirmed that the MAPK inhibitor BIRB796 suppressed the expression of the autophagy marker LC3B in HLECs under high glucose and resveratrol treatment (Fig. 4B). These results demonstrated the role of resveratrol in promoting the autophagy process in HLECs under high glucose conditions, which was mediated by the activation of the p38 MAPK signaling pathway. 
A

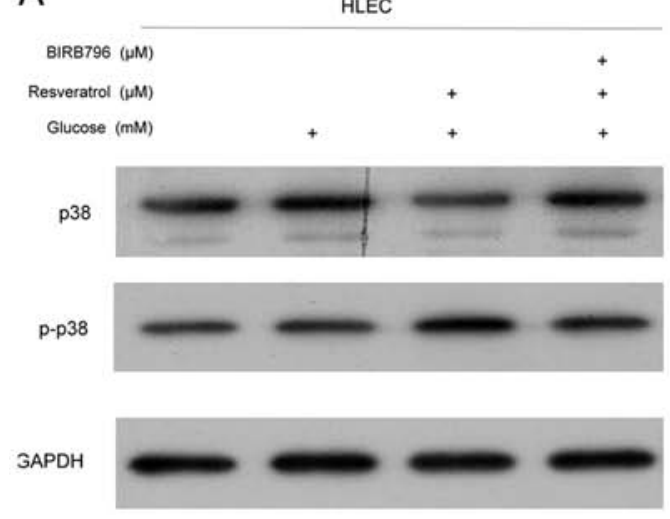

B

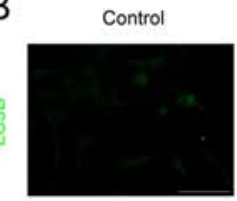

$\overline{\mathrm{a}} \mathrm{s}$

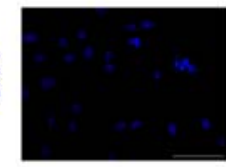

๖్

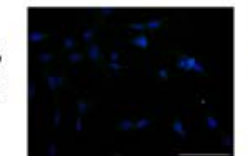

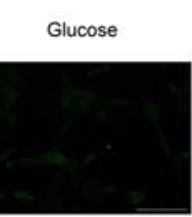
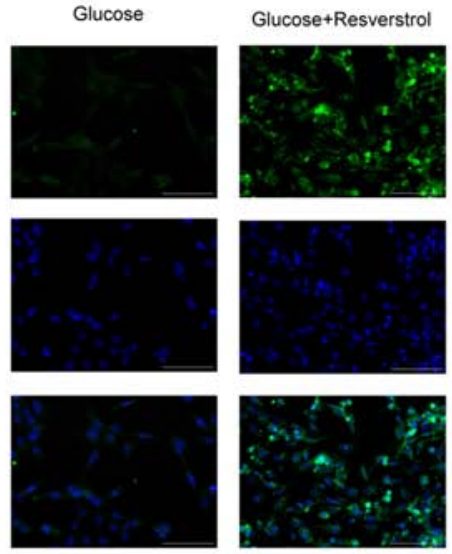
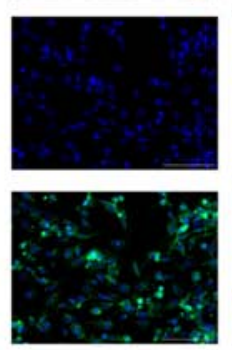

BIRB796 Glucose+Resverstrol
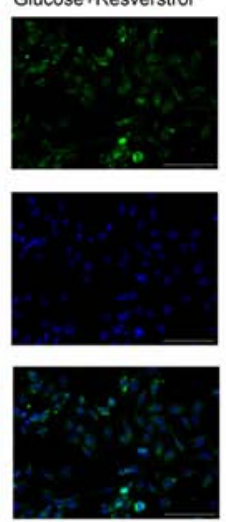

Figure 4. Resveratrol activates the p38 MAPK pathway to induce autophagy in HLECs under high glucose conditions. (A) The levels of total and phosphorylated p38 protein in HLECs treated with $25 \mathrm{mM}$ glucose, $2 \mu \mathrm{M}$ resveratrol and $10 \mu \mathrm{M}$ MAPK inhibitor BIRB796 for $24 \mathrm{~h}$ were detected by western blotting. GAPDH was used as the internal control. (B) Immunofluorescence of the expression of LC3B in HLECs treated with $25 \mathrm{mM}$ glucose, $2 \mu \mathrm{M}$ resveratrol and $10 \mu \mathrm{M}$ MAPK inhibitor BIRB796 for $24 \mathrm{~h}$. Cell nuclei were stained with DAPI. HLEC, human lens epithelial cell; LC3B, microtubule-associated protein 1 light chain $3 \beta$; MAPK, mitogen-activated protein kinase; $\mathrm{p}-$, phosphorylated.

\section{Discussion}

Due to the crucial role of high glucose-induced oxidative damage in the pathogenesis of diabetic cataract, effective reagents capable of inhibiting HLEC oxidative damage have been considered to be potential candidates for diabetic cataract prevention and treatment (12-14). Resveratrol, which is derived from grape seeds, has been established as a potent antioxidant in various contexts, such as inflammation and diabetes mellitus (20-22); however, its effects on diabetic cataract remain unclear. In the present study, it was hypothesized that resveratrol suppressed diabetic cataract pathogenesis by regulating HLEC autophagy, which was tested using high glucose-treated HLECs as an in vitro model. The results of the present study demonstrated that resveratrol effectively reversed the high glucose-induced suppression of HLEC viability. In addition, the high-glucose-induced oxidative stress and proliferation inhibition in HLECs were significantly repressed by resveratrol. Using multiple experimental assays, the present study demonstrated that resveratrol treatment promoted autophagy in HLECs under high glucose conditions, which was mediated by the resveratrol-induced activation of the p38 MAPK signaling pathway. The molecular mechanisms of the effects pf resveratrol in HLECs were finally confirmed using the MAPK inhibitor BIRB796. Taken together, the results of the present study characterized resveratrol as a new drug candidate for diabetic cataract prevention and treatment, which effectively suppressed high glucose-induced oxidative damage by activating the p38 MAPK signaling to promote autophagy in HLECs.

Natural chemical products have been established as a valuable resource of novel therapeutic drugs for human disorders. For instance, artemisinin, isolated from Artemisia annua L., has been explored as a life-saving anti-malaria drug (27) and has also been reported to possess clinical values for other human pathogenic conditions such as obesity and acute myeloid leukemia $(28,29)$. A number of such natural products have exhibited such multi-faceted roles in human disease therapy, which has also been observed to apply to resveratrol. Resveratrol was first identified as an antioxidative reagent, which possessed additional biological functions such as atherosclerosis prevention, hepatic apolipoprotein and lipid synthesis modulation (28). In addition, resveratrol has been reported to exert therapeutic effects on various human disorders such as cancer, vascular disease, neurodegenerative and metabolic diseases (30). The present study demonstrated for the first time that resveratrol effectively suppressed the inhibition of HLEC viability and oxidative stress induced by high glucose treatment, indicating its application potential as a new therapeutic drug for diabetic cataract treatment. A previous study has reported that resveratrol represses diabetes progression by modulating insulin action and pancreatic $\beta$ cell function (20). The results of the present study further highlighted the value of resveratrol as an inhibitor for diabetes mellitus and its complications.

The association of autophagy with human pathogenic conditions including diabetes mellitus have been a research focus during the past two decades, identifying autophagy as promising target for drug development (31). Autophagy in pancreatic $\beta$ cells is also associated with diabetes mellitus development and progression (32). For instance, the knockout of autophagy-related protein (ATG) 7 gene specifically in $\beta$ cells causes reduced $\beta$-cell mass and decreased pancreatic insulin synthesis and secretion, impaired response to obesity-induced ER stress, as well as hypoinsulinemia and hyperglycemia symptoms in a mouse model (33). However, the association between autophagy in LECs and diabetic cataract formation remains unclear. In the present study, resveratrol promoted autophagy in HLECs under high glucose conditions, which mediated its anti-diabetic cataract functions. LC3II protein is produced by the binding of soluble LC3I with lipid components during autophagosome formation (34). Beclin-1 is a component of the class III phosphoinositide 3-kinase complex and performs key roles in the induction of the autophagy process as a cytoprotective mechanism (35). In addition, p62 protein has been characterized as a ubiquitinand LC3-binding protein that undergoes selective degradation in response to autophagy (36). The results of the present study 
demonstrated that the protein levels of LC3II and beclin-1 were increased during resveratrol-induced autophagy in HLECs compared with normal glucose levels, accompanied by a significant decrease in the levels of LC3I and p62. These results suggested an autophagy-promoting role for resveratrol in HLECs during diabetic cataract development, and thus revealed HLEC autophagy as a promising target for new anti-diabetic cataract drug screening.

The signaling pathway responsible for modulating autophagy under high glucose conditions in HLECs was also explored in the present study. A previous study has demonstrated that autophagy formation is regulated by the p38 MAPK signaling pathway, which is activated by phosphorylation and binds with the p38-interacting protein to modulate ATG9 protein activity during autophagosome formation (37). In the present study, the phosphorylation of $\mathrm{p} 38$ protein was promoted by resveratrol in HLECs under high glucose conditions, which indicated that resveratrol-induced HLEC autophagy was mediated by the p38 MAPK and its associated signaling pathways. Notably, a previous study has also demonstrated that a number of biological roles of resveratrol were mediated by the activation of the NAD ${ }^{+}$-dependent deacetylase SIRT1 (38). In addition, a recent study has reported that SIRT1 was associated with LEC proliferation and apoptosis in a mouse model of diabetic cataract (39). The involvement of SIRT1 and other signaling pathways in the HLEC oxidative damage-inhibiting roles of resveratrol require further investigation.

In summary, the results of the present study demonstrated that the plant-derived chemical resveratrol inhibited high glucose-induced oxidative damage in HLECs, which was mediated by the activation of autophagy through modulating the p38 MAPK signaling pathway. These results suggested that resveratrol may be a promising candidate anti-diabetic cataract drug and provided a basis for its application in diabetic cataract prevention and treatment.

\section{Acknowledgements}

Not applicable.

\section{Funding}

This study was supported by The Inner Mongolia Autonomous Region Natural Science Fund Project (grant no. 2018LH08064).

\section{Availability of data and materials}

The datasets used and/or analyzed during the current study are available from the corresponding author on reasonable request.

\section{Authors' contributions}

PC designed the study. PC, ZY and ZH performed the experiments. ZY and ZH performed data analysis and interpretation. PC wrote the manuscript. All authors read and approved the final manuscript.

\section{Ethics approval and consent to participate}

Not applicable.

\section{Patient consent for publication}

Not applicable.

\section{Competing interests}

The authors declare that they have no competing interests.

\section{References}

1. Becker C, Schneider C, Aballéa S, Bailey C, Bourne R, Jick S and Meier C: Cataract in patients with diabetes mellitus-incidence rates in the UK and risk factors. Eye (Lond) 32: 1028-1035, 2018.

2. Wong IYH, Ni MY, Wong IOL, Fong N and Leung GM: Saving sight in China and beyond: The lifeline express model. BMJ Glob Health 3: e000766, 2018.

3. Ferraiolo A, Bordone C, Ramone A, Gorlero F, Gustavino C and Cordera R: Universal versus risk factor screening for gestational diabetes mellitus. Clin Exp Obstet Gynecol 45: 53-57, 2018.

4. Pollreisz A and Schmidt-Erfurth U: Diabetic cataractpathogenesis, epidemiology and treatment. J Ophthalmol 2010: 608751, 2010.

5. Šimunović M, Paradžik M, Škrabić R, Unić I, Bućan K and Škrabić V: Cataract as early ocular complication in children and adolescents with type 1 diabetes mellitus. Int J Endocrinol 2018: 6763586, 2018.

6. Obrosova IG, Chung SS and Kador PF: Diabetic cataracts: Mechanisms and management. Diabetes Metab Res Rev 26: 172-180, 2010.

7. Snow A, Shieh B, Chang KC, Pal A, Lenhart P, Ammar D, Ruzycki P, Palla S, Reddy GB and Petrash JM: Aldose reductase expression as a risk factor for cataract. Chem Biol Interact 234: 247-253, 2015.

8. Bhadada SV, Vyas VK and Goyal RK: Protective effect of Tephrosia purpurea in diabetic cataract through aldose reductase inhibitory activity. Biomed Pharmacother 83: 221-228, 2016.

9. Kim J, Kim CS, Sohn E, Lee YM, Jo K and Kim JS: Litsea japonica extract inhibits aldose reductase activity and hyperglycemia-induced Lenticular Sorbitol accumulation in $\mathrm{db} / \mathrm{db}$ mice. Evid Based Complement Alternat Med 2015: 747830, 2015.

10. Kim J, Kim CS, Sohn E, Kim H, Jeong IH and Jin SK: Lens epithelial cell apoptosis initiates diabetic cataractogenesis in the Zucker diabetic fatty rat. Graefes Arch Clin Exp Ophthalmol 248: 811-818, 2010.

11. Zhu L, Zhao K and Lou D: Apoptosis factors of lens epithelial cells Responsible for cataractogenesis in vitrectomized eyes with silicone oil tamponade. Med Sci Monit 22: 788-796, 2016.

12. Kubota M, Shui YB, Liu M, Bai F, Huang AJ, Ma N, Beebe DC and Siegfried CJ: Mitochondrial oxygen metabolism in primary human lens epithelial cells: Association with age, diabetes and glaucoma. Free Radic Biol Med 97: 513-519, 2016.

13. Gong W, Zhu G, Li J and Yang X: LncRNA MALAT1 promotes the apoptosis and oxidative stress of human lens epithelial cells via p38MAPK pathway in diabetic cataract. Diabetes Res Clin Pract 144: 314-321, 2018.

14. Nambu H, Kubo E, Takamura Y, Tsuzuki S, Tamura M and Akagi Y: Attenuation of aldose reductase gene suppresses high-glucose-induced apoptosis and oxidative stress in rat lens epithelial cells. Diabetes Res Clin Pract 82: 18-24, 2008.

15. Barlow AD and Thomas DC: Autophagy in diabetes: $\beta$-Cell dysfunction, insulin resistance, and complications. DNA Cell Bio34: 252-260, 2015.

16. Jegal KH, Ko HL, Sang MP, Byun SH, Kang KW, Cho IJ and Kim SC: Eupatilin induces Sestrin2-dependent autophagy to prevent oxidative stress. Apoptosis 21: 642-656, 2016.

17. Xie W, Zhang L, Jiao H, Guan L, Zha J, Li X, Wu M, Wang Z, Han J and You H: Chaperone-mediated autophagy prevents apoptosis by degrading BBC3/PUMA. Autophagy 11: 1623-1635, 2015.

18. Lim H, Lim YM, Kim KH, Jeon YE, Park K, Kim J, Hwang HY, Lee DJ, Pagire H, Kwon HJ, et al: A novel autophagy enhancer as a therapeutic agent against metabolic syndrome and diabetes. Nat Commun 9: 1438, 2018.

19. Lu Z, Rong $\mathrm{C}$ and Huang Y: MiR-30a inhibits BECN1-mediated autophagy in diabetic cataract. Oncotarget 8: 77360-77368, 2017. 
20. Öztürk E, Arslan AKK, Yerer MB and Bishayee A: Resveratrol and diabetes: A critical review of clinical studies. Biomed Pharmacother 95: 230-234, 2017.

21. Rodrigo R, Miranda A and Vergara L: Modulation of endogenous antioxidant system by wine polyphenols in human disease. Clin Chim Acta 412: 410-424, 2011.

22. Vinod BS, Nair HH, Vijayakurup V, Shabna A, Shah S Krishna A, Pillai KS, Thankachan S and Anto RJ: Resveratrol chemosensitizes HER-2-overexpressing breast cancer cells to docetaxel chemoresistance by inhibiting docetaxel-mediated activation of HER-2-Akt axis. Cell Death Discov 1: 15061, 2015.

23. Guo H, Chen Y, Liao L and Wu W: Resveratrol protects HUVECs from oxidized-LDL induced oxidative damage by autophagy upregulation via the AMPK/SIRT1 pathway. Cardiovasc Drugs Ther 27: 189-198, 2013.

24. Wang J, Li J, Cao N, Li Z, Han J and Li L: Resveratrol, an activator of SIRT1, induces protective autophagy in non-small-cell lung cancer via inhibiting Akt/mTOR and activating p38-MAPK. OncoTargets Ther 11: 7777-7786, 2018.

25. Wu Z, Yin X, Ji L, Gao YY, Pan YM, Lu Q and Wang JY: Ginkgo biloba extract prevents against apoptosis induced by high glucose in human lens epithelial cells. Acta Pharmacol Sin 29: 1042-1050, 2008.

26. He Y, She H, Zhang T, Xu H, Cheng L, Yepes M, Zhao Y and Mao Z: p38 MAPK inhibits autophagy and promotes microglial inflammatory responses by phosphorylating ULK1. J Cell Biol 217: 315-328, 2018.

27. Fairhurst RM, Nayyar GM, Breman JG, Hallett R Vennerstrom JL, Duong S, Ringwald P, Wellems TE, Plowe CV and Dondorp AM: Artemisinin-resistant malaria: Research challenges, opportunities, and public health implications. Am J Trop Med Hyg 87: 231-241, 2012.

28. Drenberg CD, Buaboonnam J, Orwick SJ, Hu S, Li L, Fan Y, Shelat AA, Guy RK, Rubnitz J and Baker SD: Evaluation of artemisinins for the treatment of acute myeloid leukemia. Cancer Chemother Pharmacol 77: 1231-1243, 2016.

29. Ping L, Zhang F, Qian S, Li X, Cui ZM, Dang YJ and Tang QQ: Artemisinin derivatives prevent obesity by inducing browning of WAT and enhancing BAT function. Cell Res 26: 1169-1172, 2016.
30. Kuršvietienè L, Stanevičienè I, Mongirdienè A and Bernatonienè J: Multiplicity of effects and health benefits of resveratrol. Medicina (Kaunas) 52: 148-155, 2016.

31. Gao L, Jauregui CE and Teng Y: Targeting autophagy as a strategy for drug discovery and therapeutic modulation. Future Med Chem 9: 335-345, 2017.

32. Marasco MR and Linnemann AK: $\beta$-cell autophagy in diabetes pathogenesis. Endocrinology 159: 2127-2141, 2018.

33. Quan W, Lim YM and Lee MS: Role of autophagy in diabetes and endoplasmic reticulum stress of pancreatic $\beta$-cells. Exp Mol Med 44: 81-88, 2012.

34. Mcleland CB, Rodriguez J and Stern ST: Autophagy monitoring assay: Qualitative analysis of MAP LC3-I to II conversion by immunoblot. Methods Mol Biol 697: 199, 2011.

35. Pattingre S, Tassa A, Qu X, Garuti R, Liang XH, Mizushima N, Packer M, Schneider MD and Levine B: Bcl-2 antiapoptotic proteins inhibit Beclin 1-dependent autophagy. Cell 122: 927-939, 2005

36. Komatsu $\mathrm{M}$ and Ichimura Y: Physiological significance of selective degradation of p 62 by autophagy. FEBS Lett 584: 1374-1378, 2010.

37. Guo F, He XB, Li S and Le W: A central role for phosphorylated $\mathrm{p} 38 \alpha$ in linking proteasome inhibition-induced apoptosis and autophagy. Mol Neurobiol 54: 1-13, 2016.

38. Chung S, Yao H, Caito S, Hwang JW, Arunachalam G and Rahman I: Regulation of SIRT1 in cellular functions: Role of polyphenols. Arch Biochem Biophys 501: 79-90, 2010.

39. Zeng K, Feng QG, Lin BT, Ma DH and Liu CM: Effects of microRNA-211 on proliferation and apoptosis of lens epithelial cells by targeting SIRT1 gene in diabetic cataract mice. Bioscience Reports 37: BSR20170695, 2017.

(2) (1) () This work is licensed under a Creative Commons

Attribution-NonCommercial-NoDerivatives 4.0 International (CC BY-NC-ND 4.0) License. 